\title{
Correction to: Effect of reduced opportunities on bargaining outcomes: an experiment with status asymmetries
}

\author{
Subrato Banerjee ${ }^{1,2}$ \\ Published online: 8 June 2020 \\ (C) Springer Science+Business Media, LLC, part of Springer Nature 2020
}

\section{Correction to: Theory and Decision https://doi.org/10.1007/s11238- 020-09754-4}

Part of the equation in sub-section "Key findings" is missing in the original publication of the article. The error was caused by the fact that the equation, due to its length, exceeded the page width. The missing part of the equation is given below:

The right-hand side equals:

$$
\begin{aligned}
& \alpha_{1} \operatorname{RankBarg}_{i} \cdot \Delta^{j} \operatorname{RelPos}_{j}+\alpha_{2} \operatorname{RandmContr}_{i} \cdot \Delta^{j} \operatorname{RelPos}_{j}+\alpha_{3} \operatorname{RankContr}_{i} \\
& \cdot \Delta^{j} \operatorname{RelPos}_{j}+\left(\Delta^{j} \boldsymbol{X}_{i j}\right) \boldsymbol{\beta}+\Delta^{j} \varepsilon_{i j} .
\end{aligned}
$$

The original article has been updated.

The original article can be found online at https://doi.org/10.1007/s11238-020-09754-4.

\section{Subrato Banerjee}

subrato.banerjee@unimelb.edu.au

1 Queensland University of Technology Business School (Centre for Behavioural Economics, Society and Technology), Brisbane, QLD, Australia

2 University of Melbourne (Australia India Institute), Melbourne, VIC, Australia 